\title{
Unnecessary PCI Attempt for Presumed CTO Which Was Revealed To Be Anomalous Coronary Arteries - Role of Coronary CT Angiography
}

\author{
Hyungdon Kook, MD, PhD, ${ }^{1}$ Jin-Ho Choi, MD, PhD, ${ }^{2}$ Hyun Jong Lee, MD, ${ }^{3}$ Cheol Woong Yu, MD, PhD ${ }^{1}$ \\ ${ }^{1}$ Division of Cardiology, Cardiovascular Center, Korea University Anam Hospital, Seoul, Korea; ${ }^{2}$ Division of Cardiology, Department \\ of Internal Medicine, Sungkyunkwan University School of Medicine, Seoul, Korea; ${ }^{3}$ Division of Cardiology, Department of Internal \\ medicine, Sejong General Hospital, Bucheon-si, Gyeonggi-do, Korea
}

\section{ABSTRACT}

Herein, we present a brief case of anomalous coronary arteries mistaken to be chronic total occlusion. Since we first presumed the anomalous coronary arteries to be chronic total occlusion of the distal right coronary artery, percutaneous coronary intervention was attempted for the presumed lesion, but it failed. Before the second attempt of percutaneous coronary intervention, coronary computed tomography angiography revealed the coronary artery from the left anterior descending artery corresponding with the distal part of the right coronary artery without connection to the right coronary artery. Therefore, we recognized the patient had anomalous coronary arteries with no connection of the vascular wall between the main and distal segments of the right coronary artery.

This case may give insights to the importance of meticulous examination of coronary computed tomography angiography imaging before chronic total occlusion percutaneous coronary intervention to avoid the unnecessary procedure.

\section{INTRODUCTION}

Coronary computed tomography angiography (CCTA) to aid with pre-procedural planning before chronic total occlusion (CTO) percutaneous coronary intervention (PCI) is not only helpful with understanding the CTO anatomy, but it also is known to increase the success rate of the CTO PCI [Brilakis 2019; Yu 2017]. However, due to the economic cost and time-consuming issues with CCTA, a considerable number of CTO PCI still is being performed without CCTA guidance in real-world practice. Herein, we present a brief case about a failed PCI attempt for a presumed CTO lesion, which was revealed to be anomalous coronary artery on CCTA.

Received fune 15, 2020; accepted fuly 17, 2020.

Correspondence: Cheol Woong Yu, MD, PhD, Cardiovascular Center (Interventional Cardiology), Anam Hospital, Korea University Medical Center, 126-1, Anam-dong 5ga, Seongbuk-gu, Seoul, 136-705, Republic of Korea; +82-10-7266-3389; fax +82-2-927-1478 (e-mail: ycw717@naver.com).

\section{CASE REPORT}

A 50-year-old male patient presented with stable angina. The patient had hypertension as an underlying disease and a family history of coronary artery disease. Treadmill test showed horizontal ST-segment depression in the inferior and lateral leads on the electrocardiogram, during the exercise phase and down sloping ST-segment depression in the same leads during the recovery phase. An absence of coronary artery segment corresponding with the distal right coronary artery (RCA) was shown in angiography, and the left anterior descending (LAD) coronary artery was connected to
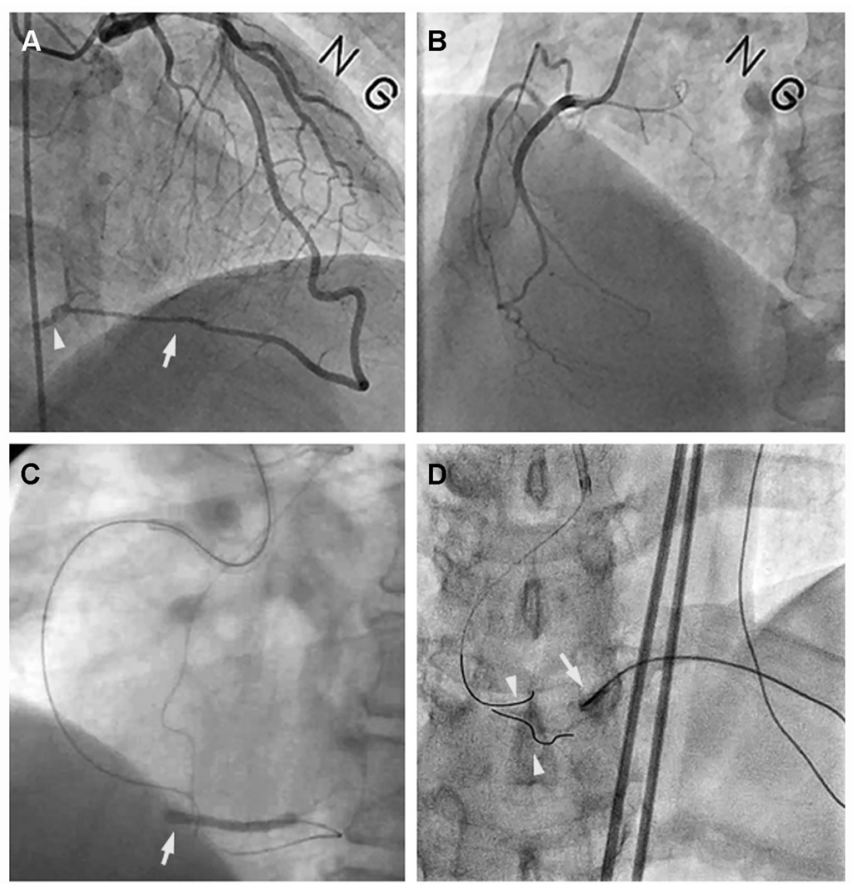

Figure 1. Coronary angiography and percutaneous coronary intervention. A) Coronary angiography showing left anterior descending coronary artery connected to posterior descending artery (arrow) and presumed distal RCA (arrowhead). B) Hypoplastic RCA. C) Percutaneous coronary intervention using retrograde approach. The presumed distal RCA and blockade of proximal segment (arrow) is visualized. D) Two intracoronary guidewires (arrowheads) and microcatheter (arrow). RCA: right coronary artery 

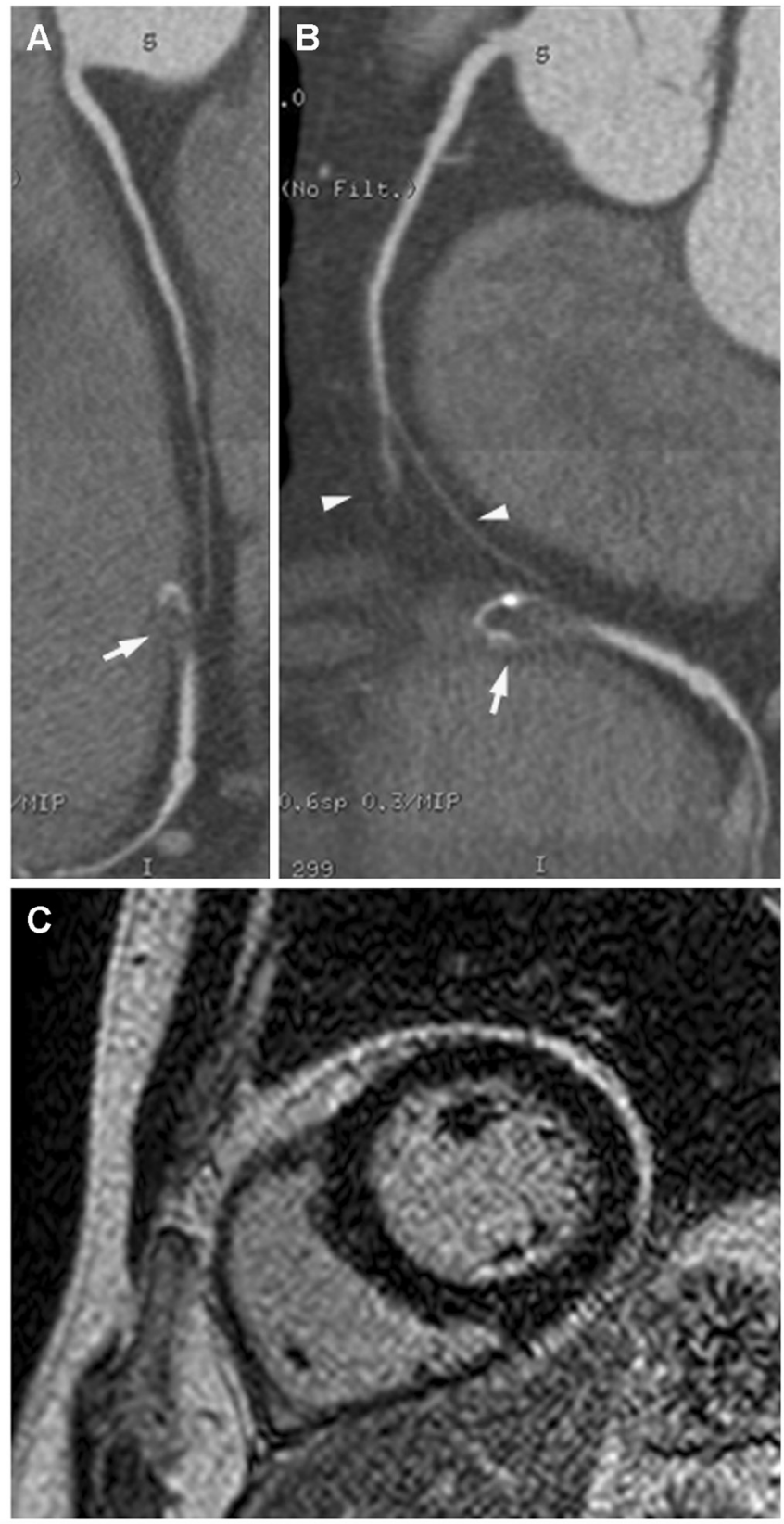

Figure 2. Coronary computed tomography angiography and cardiac magnetic resonance imaging. A, B) The end of coronary artery continuous from left anterior descending coronary artery was surrounded by calcified wall and distal cap (arrows). Notice two arrowheads indicating small branches of hypoplastic right coronary. C) No late gadolinium enhancement in left ventricular myocardium by cardiac magnetic resonance imaging.

the posterior descending artery and posterolateral branches (Figures 1A and 1B). The posterior descending artery and posterolateral branches connected with the LAD seemed to compete with the distal flow of the RCA, through which we assumed there would be CTO of the distal RCA. Another reason to suspect the CTO of the distal RCA was that the distal end of the posterior descending artery and posterolateral branches connecting to the LAD seemed to be abruptly disconnected. PCI for the presumed CTO was attempted with retrograde approach, but it failed (Figures $1 \mathrm{C}$ and $1 \mathrm{D}$ ). No wire could cross the presumed occluded segment. CCTA before the second PCI attempt revealed coronary artery corresponding to the distal RCA without connection to the RCA. The end of the coronary artery continuous from the LAD coronary artery was surrounded by calcified wall and a distal cap. There was no connection of the vascular wall between the right coronary artery and presumed posterior descending artery in CCTA images [Choi 2011] (Figures 2A and 2B). Cardiac magnetic resonance imaging showed a normal regional wall motion and no late gadolinium enhancement in the left ventricular myocardium. There is digital content that demonstrates cardiac magnetic resonance two-chamber view cine movie with no regional wall motional abnormality [Choi 2013] (Figure 2C). Therefore, we recognized the patient had anomalous coronary arteries with no connection of the vascular wall between the main and distal segments of the RCA.

After coronary anomaly was confirmed by CCTA and cardiac magnetic resonance imaging showed normal regional wall motion without late left ventricular gadolinium enhancement, we estimated that the cause of ischemia in the patient was microvascular angina or ischemia induced by the coronary artery anomaly itself. The symptoms improved after medical therapy consisting of nicorandil, trimetazidine, and statin.

\section{DISCUSSION}

With the development of dedicated devices and new techniques, CTO PCI has made significant progress in recent years [Rathore 2009]. Among them, CCTA and imaging reconstruction technology has markedly contributed to the understanding of CTO lesion anatomy. On the other hand, if CCTA routinely is implemented before the CTO PCI, there is a risk that patients will be exposed to more contrast agents and radiation. The additional economic burden and time required for coronary artery imaging reconstruction also are not negligible. Also, clinical presentation of an angina patient can be an important factor with deciding whether to implement CCTA before PCI. For these reasons, a considerable number of CTO PCIs still are being performed without pre-procedural CCTA evaluation in real-world practice. However, when evaluating a CTO lesion using only coronary angiography, it may be difficult to distinguish between CTO and subtotal occlusion, and rarely, it may not be possible to distinguish it from anomalous coronary arteries that resemble CTO as introduced in this case report [Choi 2015]. The estimated prevalence of coronary artery anomalies varies, ranging from $0.21 \%$ to $6 \%$ based on angiography, CCTA, and autopsy databanks [Perez-Pomares 2016; Aydinlar 2005]. An anomalous origin of posterior descending artery and posterolateral branches from LAD, one of the anomalies of intrinsic coronary artery anatomy, is a very rare coronary artery anomaly (prevalence of 0 to $0.028 \%$ ) and some of this anomaly 
might be only confirmed with CCTA [Yildiz 2010; Cheng 2010]. Despite the remarkable development of CTO PCI, the success rate still is lower, and the incidence of complications is higher than that of conventional PCI [Hannan 2016; Brilakis 2015]. Therefore, the benefits of pre-procedural meticulous CCTA image analysis to avoid unnecessary PCI procedures may outweigh its drawbacks.

\section{REFERENCES}

Aydinlar A, Cicek D, Senturk T, et al. 2005. Primary congenital anomalies of the coronary arteries: a coronary arteriographic study in Western Turkey. International heart journal. 46(1):97-103.

Brilakis ES, Banerjee S, Karmpaliotis D, et al. 2015. Procedural outcomes of chronic total occlusion percutaneous coronary intervention: a report from the NCDR (National Cardiovascular Data Registry). JACC Cardiovasc Interv. 8(2):245-253.

Brilakis ES, Mashayekhi K, Tsuchikane E, et al. 2019. Guiding Principles for Chronic Total Occlusion Percutaneous Coronary Intervention. Circulation. 140(5):420-433.

Cheng Z, Wang X, Duan Y, et al. 2010. Detection of coronary artery anomalies by dual-source CT coronary angiography. Clinical radiology. 65(10):815-822.

Choi JH, Chang SA, Choi JO, et al. 2013. Frequency of myocardial infarction and its relationship to angiographic collateral flow in territories supplied by chronically occluded coronary arteries. Circulation. 127(6):703-709.
Choi JH, Kim EK, Kim SM, et al. 2015. Noninvasive Discrimination of Coronary Chronic Total Occlusion and Subtotal Occlusion by Coronary Computed Tomography Angiography. JACC Cardiovasc Interv. 8(9):1143-1153.

Choi JH, Song YB, Hahn JY, et al. 2011. Three-dimensional quantitative volumetry of chronic total occlusion plaque using coronary multidetector computed tomography. Circ J. 75(2):366-375.

Hannan EL, Zhong Y, Jacobs AK, et al. 2016. Patients With Chronic Total Occlusions Undergoing Percutaneous Coronary Interventions: Characteristics, Success, and Outcomes. Circ Cardiovasc Interv. 9(5):e003586.

Perez-Pomares JM, de la Pompa JL, Franco D, et al. 2016. Congenital coronary artery anomalies: a bridge from embryology to anatomy and pathophysiology--a position statement of the development, anatomy, and pathology ESC Working Group. Cardiovasc Res. 109(2):204-216.

Rathore S, Matsuo H, Terashima M, et al. 2009. Procedural and in-hospital outcomes after percutaneous coronary intervention for chronic total occlusions of coronary arteries 2002 to 2008: impact of novel guidewire techniques. JACC Cardiovasc Interv. 2(6):489-497.

Yildiz A, Okcun B, Peker T, Arslan C, Olcay A, Bulent Vatan M. 2010. Prevalence of coronary artery anomalies in 12,457 adult patients who underwent coronary angiography. Clin Cardiol. 33(12):E60-64.

Yu CW, Lee HJ, Suh J, et al. 2017. Coronary Computed Tomography Angiography Predicts Guidewire Crossing and Success of Percutaneous Intervention for Chronic Total Occlusion: Korean Multicenter CTO CT Registry Score as a Tool for Assessing Difficulty in Chronic Total Occlusion Percutaneous Coronary Intervention. Circ Cardiovasc Imaging. 10(4). 